\title{
Le matériel et les exigences associées
}

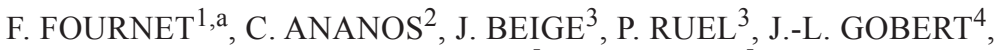 \\ J.-M. CHAMPAGNE ${ }^{5}$, J.-C. GODIN ${ }^{5}$, \\ S. ISRAËL ${ }^{5}$, P. MOREAU ${ }^{6}$, G. ABÉLA $^{7}$, P. BONAVENTURE $^{8}$, \\ V. DIDIER ${ }^{8}$, J. FAZILEABASSE$^{9}$
}

\begin{abstract}
RÉSUMÉ Cet atelier, créé dans le cadre du GT SFRP-COFREND sur la radiographie industrielle a étudié le matériel utilisé en France. Ont été décrits les différents types de matériel, les générateurs de rayons $X$ et les appareils contenant des sources radioactives. Les accessoires, et matériels de délimitation et systèmes de collimation du faisceau sont aussi décrits. Pour chaque type de matériel, des axes d'améliorations et propositions d'amélioration sont cités.
\end{abstract}

ABSTRACT Industrial radiographic devices and associate requirements.

This workshop, created within the framework of WP SFRP-COFREND on industrial radiography studied the material used in France for industrial radiography. Were described the various types of material, the generators of $\mathrm{X}$-rays and the apparatuses containing of the radioactive sources. Accessories, and materials of delimitation and systems of collimation of the beam are also described. For each type of material, axes of improvements and proposals for an improvement are quoted.

Keywords: radiographie industrielle / matériel / collimateur / balisage

\section{Introduction}

Les contrôles non destructifs basés sur les propriétés des rayonnements ionisants peuvent être réalisés par deux familles de matériel.

\subsection{Les appareils électriques générateurs de rayons $X$}

Ils sont couramment utilisés pour contrôler des pièces métalliques de « faible » épaisseur ou constituées de matériaux composites ou plastiques. La haute tension appliquée au tube radiogène peut atteindre $420 \mathrm{kV}$ pour les appareils les plus puissants.

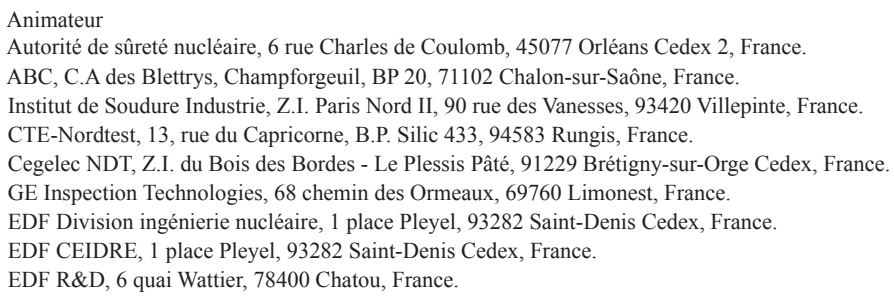



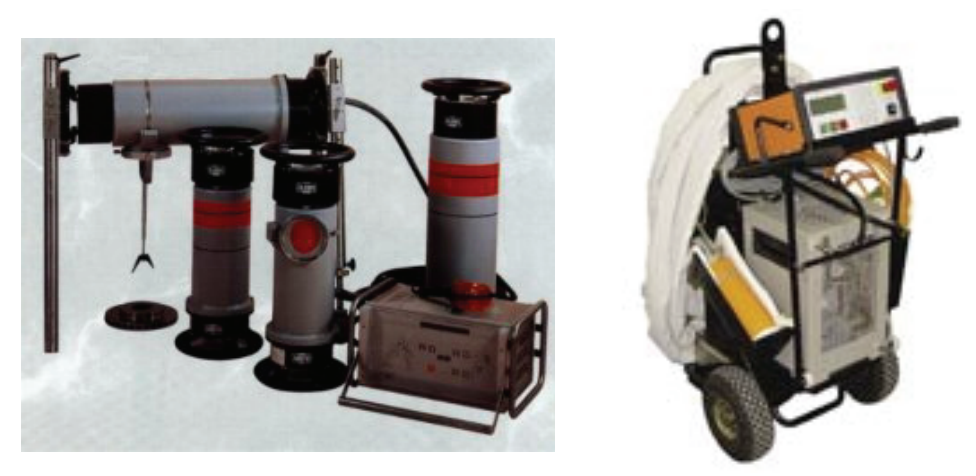

Figure 1 - Générateurs de rayons $X$.

Generators of $X$-rays.

Mais la plupart sont lourds (l'ensemble intégré tube radiogène + générateur haute tension peut atteindre quelques dizaines de $\mathrm{kg}$ ), encombrants et nécessitent une alimentation électrique. Leur mise en œuvre sur chantiers n'est donc pas toujours aisée : on les retrouve ainsi pour une grande part utilisés à poste fixe dans des enceintes dédiées (local ou cabine).

Des générateurs de rayons X (Fig. 1) sont destinés au contrôle en ligne des soudures lors de la pose de canalisations (exposition panoramique endotubulaire) : on les appelle des « crawlers ». Il s'agit en fait d'un tube radiogène alimenté par batteries et associé à un chariot automoteur.

Des accélérateurs d'électrons sont également ponctuellement utilisés pour des contrôles non destructifs très spécifiques.

Du fait de la mise en place tardive d'un système d'autorisation administrative préalable à leur utilisation (depuis 2002), il est aujourd'hui encore difficile de connaître de manière exhaustive le nombre et les types d'appareils électriques générateurs de rayons X utilisés en France. Toutefois, depuis novembre 2007, le Code de la santé publique a également instauré un régime d'autorisation pour pouvoir distribuer ce type de matériel : cela permettra sans aucun doute d'avoir une meilleure visibilité à moyen terme du parc français.

\subsection{Les appareils contenant une source radioactive scellée}

Leur principe repose sur les propriétés d'atténuation par le matériau traversé du rayonnement gamma émis par une source radioactive. Ils sont ainsi couramment appelés gammagraphes ou projecteurs gammagraphiques. 


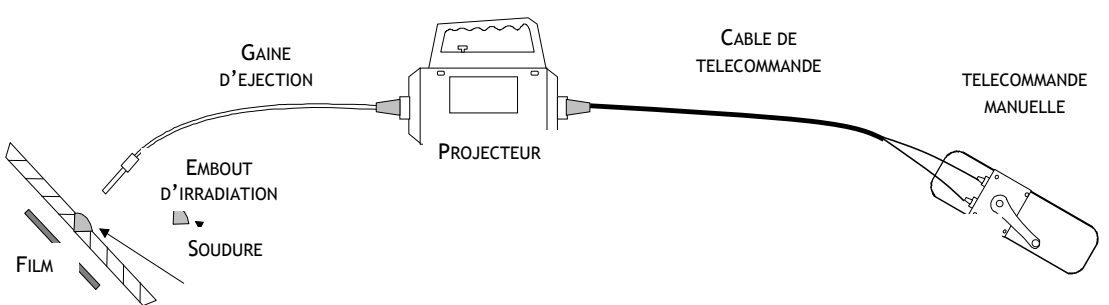

Figure 2 - Schéma simplifié d'un gammagraphe ${ }^{\circledR}$ à éjection manuelle. Simplified diagram of a gammagraphe ${ }^{\circledR}$ with manual ejection.

La source radioactive scellée est placée dans un porte-source stocké au coeur du projecteur. La sortie du porte-source dans la gaine d'éjection, jusqu'au dispositif d'irradiation (embout ou collimateur), est réalisée par l'opérateur via une télécommande (manuelle ou électrique). L'exposition de l'ensemble «pièce contrôlée-film » est réalisée dans une configuration prédéfinie en fonction de la technique choisie, dépendante de la géométrie de la pièce (Fig. 2).

Les radionucléides autorisés en France, pour être utilisés à des fins de gammagraphie sont répertoriés dans le tableau I.

\section{Les matériels}

\subsection{Projecteurs gammagraphiques et accessoires}

\subsubsection{Parc existant}

Les gammagraphes utilisés en France doivent répondre depuis 1985 aux attendus du décret $n^{\circ} 85-968$ du 27 août 1985 définissant les conditions d'hygiène et de sécurité qui leur sont applicables, issues de la norme française NF M 60-551 de juin 1983.

Plusieurs éléments ont ainsi été imposés : signalisation colorée indiquant l'état du dispositif, système spécifique de verrouillage manuel par clé, fermeture automatique du système obturateur lors de la rentrée de la source, "réarmement » manuel, révision annuelle du projecteur et de ses accessoires... En comparaison avec la plupart des appareils de conception étrangère, ceux utilisés en France présentent donc intrinsèquement de plus grandes garanties quant à leur sûreté de fonctionnement et à la sécurité de leurs utilisateurs : le décret $n^{\circ} 85-968$ est en effet très contraignant. 
TABLEAU I

Les radionucléides autorisés en France.

Radionuclides authorized in France.

\begin{tabular}{|c|c|c|c|}
\hline $\begin{array}{c}\text { Radionucléide } \\
\text { (par ordre décroissant } \\
\text { d'énergie gamma moyenne) }\end{array}$ & Cobalt-60 & Iridium-192 & Sélénium-75 \\
\hline Activités maximales autorisées & $\begin{array}{c}\text { GR30 : 1,11 TBq } \\
\text { GR50:2,2 TBq } \\
\text { GMA2500:18,5 TBq }\end{array}$ & $\begin{array}{c}\text { Jusqu'à } 11,1 \mathrm{TBq} \\
\text { (appareils spécifiques) } \\
\text { GAM80 : 2,96 TBq } \\
\text { GAM120:4,44 TBq }\end{array}$ & $\begin{array}{c}2,96 \mathrm{TBq} \\
\text { (pour l'instant, } \\
\text { GAM80/120 uniquement) }\end{array}$ \\
\hline $\begin{array}{l}\text { Constante spécifique, } \\
\text { en } \mathrm{mSv}^{-1} \mathrm{~h}^{-1} \cdot \mathrm{TBq}^{-1} \text { à } 1 \mathrm{~m}^{(1)}\end{array}$ & 305 & 108 & de l'ordre de 60 \\
\hline Période radioactive & 5,27 ans & 73,8 jours & 119,8 jours \\
\hline $\begin{array}{l}\text { Principales émissions gamma, } \\
\text { en } \mathrm{keV}^{(2)}\end{array}$ & $\begin{array}{c}1333(100 \%)-\mathrm{E}_{\max } \\
1173(99,9 \%)\end{array}$ & $\begin{array}{c}316,5(83 \%) \\
468,1(47,8 \%) \\
308,5(29,8 \%) \\
612,5(5,3 \%)-E_{\max }\end{array}$ & $\begin{array}{c}264,7(59,1 \%) \\
136(59 \%) \\
279,5(25,2 \%) \\
400,7(11,6 \%)-E_{\max }\end{array}$ \\
\hline $\begin{array}{c}\text { Épaisseur moitié } \\
\text { dans le plomb, en mm }\end{array}$ & 11 & 4 & 1 \\
\hline $\begin{array}{c}\text { Épaisseur moitié } \\
\text { dans l'acier, en mm }\end{array}$ & 26 & 17 & 10 \\
\hline $\begin{array}{c}\text { Épaisseur dixième } \\
\text { dans le plomb, en mm }{ }^{(3)}\end{array}$ & 47 & 12 & 5 \\
\hline $\begin{array}{c}\text { Épaisseur dixième } \\
\text { dans l'acier, en mm }{ }^{(3)}\end{array}$ & 92 & 56 & 41 \\
\hline
\end{tabular}

(1) Pour l'Iridium et le Cobalt, les valeurs proviennent de la norme expérimentale M 62-103 de septembre 1988 (Radioprotection - Installations de radiologie gamma industrielle - Atténuation des rayonnements ionisants par les écrans de protection). Pour le sélénium, la valeur indiquée représente une moyenne de plusieurs données de la littérature.

${ }^{(2)}$ Données issues du recueil « Radionucléides » (édition de 1991), CEA DAMRI.

(3) Valeurs extrapolées des courbes présentes dans le guide pratique « Radionucléides et Radioprotection » (édition 2006), par D. Delacroix, J.P. Guerre et P. Leblanc, tenant compte des effets de build-up dans la protection.

Sur le parc français, de l'ordre de 600 gammagraphes (Fig. 3) sont utilisés. Environ $85 \%$ sont de type GAM80/120 (appareils portatifs généralement chargés à l'iridium-192), $10 \%$ de type GR30/50 (Fig. 4) et GMA2500 (appareils mobiles sur chariots, chargés au cobalt-60) (Fig. 5).

La maintenance et le rechargement de ces appareils sont assurés par la société Cegelec.

Le reste du parc est composé d'appareils portatifs de type NUCLEAR SU100VA et de quelques appareils spécifiques généralement utilisés à poste fixe dans des installations dédiées.

\section{Axes d'amélioration}

Pour les appareils GAM80/120, bien que le visa d'examen technique VET (conformité d'un type d'appareil au regard du décret n85-986, accordée après 


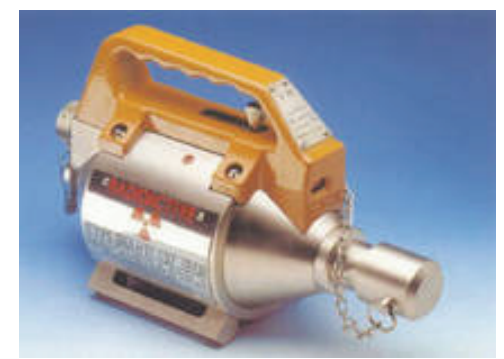

Figure 3 - GAM 80/120 ${ }^{\circledR}$. $G A M^{\circledR} 80 / 120$.

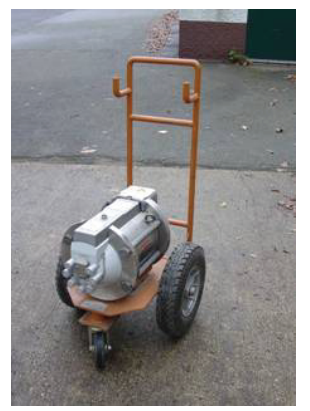

Figure $4-G R 30 / 50^{\circledR}$.

GR $30 / G R 50^{\circledR}$.

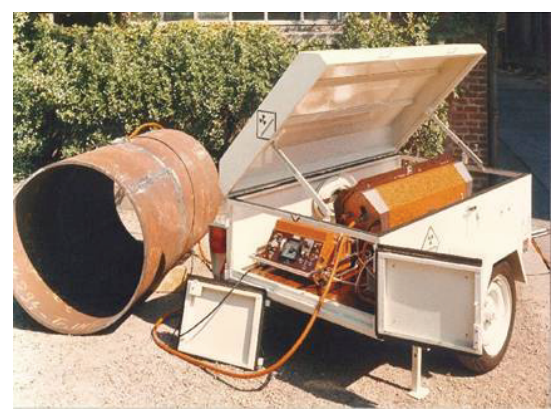

Figure 5-GMA2500 ${ }^{\circledR}$.

GMA $2500^{\circledR}$. 
investigation par le Laboratoire national de métrologie et d'essais) empêche pour le moment la modification du modèle type, quelques pistes d'amélioration ont été identifiées :

- amélioration de l'ergonomie de la poignée (section ronde au lieu de rectangulaire),

- ajout de points d'élingue amovibles de type anneau (actuellement, potentialité d'accrochage du levier d'armement en cas d'élinguage par la poignée),

- renforcement de la visibilité de la signalisation colorée (lecture difficile dans des lieux à faible luminosité ou si le projecteur est « vinylé » pour éviter la contamination radioactive).

Par ailleurs, un inconvénient est souvent mis en avant par les opérateurs : la protection radiologique en uranium appauvri induit une masse importante pour les appareils portatifs (17 kg pour un GAM80, $20 \mathrm{~kg}$ pour un GAM120). Malheureusement, aucune solution n'est pour l'instant envisageable sur le parc existant (emploi d'un autre matériau, réduction des protections radiologiques...).

Il faut cependant noter qu'une demande est actuellement en cours d'instruction par l'Autorité de sûreté nucléaire (ASN) concernant la distribution en France de l'appareil NORDION Gammamat SE (Fig. 6). Il est plus léger qu'un GAM80 : $9 \mathrm{~kg}$ en mode éjection ou $15 \mathrm{~kg}$ avec collimateur amovible intégré. Cet appareil, chargé au sélénium-75 (jusqu'à 2,96 TBq), peut en effet être utilisé sans gaine d'éjection (présence d'un collimateur intégré directionnel et panoramique).

Une expertise est en cours pour déterminer si cet appareil répond aux attendus du décret n85-968 et, dans le cas contraire, définir les modifications à lui apporter en vue de sa distribution.

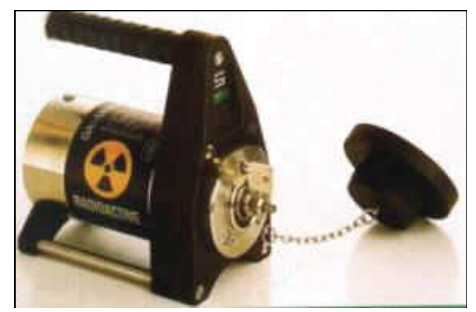

Figure 6-NORDION Gammamat $S E{ }^{\circledR}$. NORDION Gammamat SE ${ }^{\circledR}$. 


\subsubsection{Accessoires : gaines d'éjection / embout d'irradiation / télécommande}

\section{Axe d'amélioration}

L'origine de la majorité des incidents rencontrés lors de l'utilisation sur chantiers d'un gammagraphe est la présence de corps étrangers (poussières, terre, débris métalliques...) dans un des éléments de l'appareil. Cela peut en effet entraîner un retour difficile du porte-source dans le projecteur (position de sécurité) ou dans les cas extrêmes, son blocage dans la gaine d'éjection.

Dans ce cadre, les bouchons (Fig. 7) ou capuchons de protection actuels ne sont pas jugés très pratiques et peuvent parfois avoir un effet contraire à celui recherché : si l'opérateur oublie de lier les capuchons entre eux, ils peuvent traîner à même le sol et récupérer ainsi les éléments présents.

Il serait donc intéressant d'ajouter une «barrière » physique supplémentaire (clapet, membrane...) sur les embouts des gaines (notamment sur celles d'éjection, pas toujours pourvues de bouchons/capuchons) afin de limiter au maximum l'introduction d'éventuels corps étrangers.

Il est de toute façon actuellement recommandé de vérifier puis remettre en place sur les accessoires concernés, dès leur fin d'utilisation, leurs bouchons/capuchons de protection.

Par ailleurs, avant toute mise en œuvre sur chantier, il est souhaitable de faire une vérification rapide de l'état de chacun des accessoires afin de prévenir tout problème dû à la présence de corps étrangers.

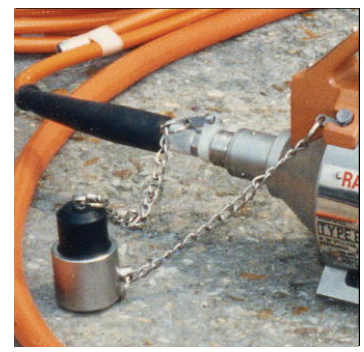

Figure 7 - Bouchons de protection.

Protection plugs. 


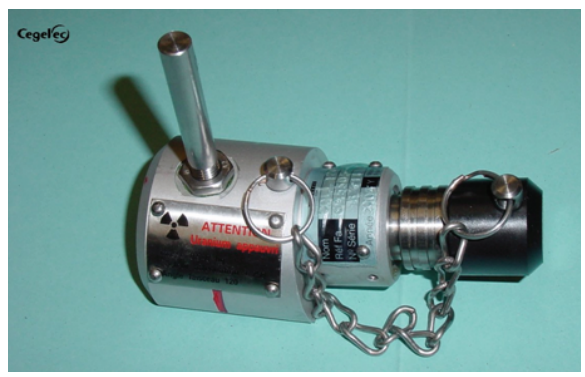

Figure 8 - Collimateur directionnel.

Directional collimator.

\subsubsection{Collimateurs en uranium appauvri}

Il existe actuellement cinq types de collimateurs :

- trois pour l'iridium-192 : atténuation $1 / 250^{\mathrm{e}}$ directionnelle (Fig. 8), atténuation $1 / 400^{\mathrm{e}}$ directionnelle ou panoramique (Fig. 9),

- deux pour le cobalt-60: atténuation $1 / 300^{\mathrm{e}}$ directionnelle (Fig. 8) ou panoramique (Fig. 9).

Cependant, certains d'entre eux ne sont plus commercialisés $\left(1 / 400^{e}\right.$ directionnelle).

Quelques centaines de collimateurs, principalement pour les modèles GAM80/120, sont utilisés en France.

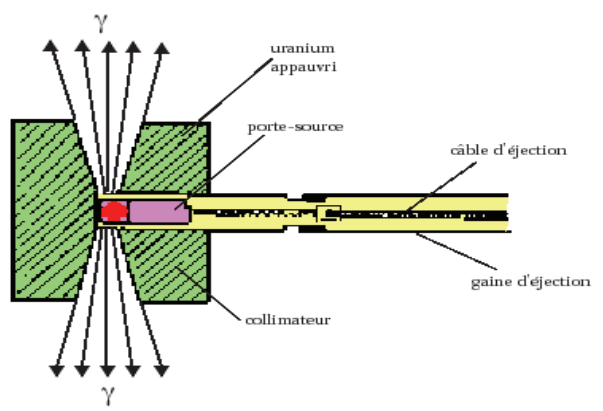

Figure 9 - Collimateur panoramique.

Panoramic collimator. 
Sur le plan de la radioprotection, leur mise en œuvre procure un avantage indéniable : l'espace couvert par le rayonnement primaire issu de la source radioactive est considérablement réduit, limitant ainsi l'ambiance radiologique autour du collimateur lors de l'exposition.

\section{Axe d'amélioration}

Le collimateur en lui-même est souvent jugé trop lourd (risques de chute lors de sa fixation, problèmes pratiques de mise en œuvre...) du fait de sa réalisation en uranium appauvri. La substitution de ce matériau par un alliage à base de tungstène, de densité légèrement moindre, représenterait une piste intéressante pour la conception d'un nouveau type de collimateur. De plus, cela permettrait de s'affranchir du suivi administratif lié à l'uranium (comptabilité des matières nucléaires / transport de matières radioactives).

Dans ce cadre, il faut souligner l'initiative locale d'EDF et de la société CICO Centre : un système de positionnement a été développé (cf. paragraphe 2.1.4) comprenant notamment un atténuateur usiné en alliage cuivre-tungstène (Fig. 10). Celui-ci se positionne directement sur l'embout d'irradiation du gammagraphe et permet un angle de collimation de $60^{\circ}$.

Bien que des mesures fines soient encore à réaliser pour connaître l'atténuation effective du rayonnement gamma lors de l'emploi de ce dispositif, il représente néanmoins une avancée intéressante dans le domaine.

\section{Propositions}

Du fait de l'avantage qu'il procure en termes de radioprotection, l'emploi systématique lors de chantiers gammagraphiques d'un dispositif de collimation ou d'atténuation du

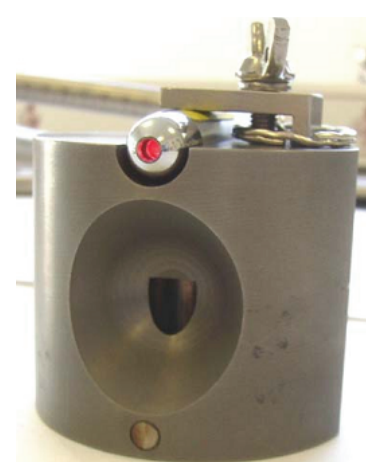

Figure 10 - Système de positionnement $\mathrm{CICO}^{\circledR}$. CICO System of positioning ${ }^{\circledR}$. 
rayonnement est recommandé, sauf impossibilités techniques avérées dues à la configuration de la pièce à contrôler (risques de chute, problèmes de positionnement...).

Les différentes procédures, codes, normes ou modes opératoires relatifs aux contrôles non destructifs devraient si nécessaires être révisés pour prendre en compte cette systématisation (révision des procédures qualifiées...).

Dans les cas où l'emploi d'un collimateur est impossible, il est préconisé d'avoir recours à des dispositifs adaptés de protection collective : protections radiologiques souples, objets spécifiquement manufacturés en plomb ou acier $(c f$. paragraphe 2.3.2)...

\subsubsection{Systèmes de positionnement}

Le placement rigoureux du dispositif d'irradiation (embout ou collimateur) par rapport à la pièce à contrôler et au film à exposer est primordial : couverture totale du champ à exposer, incidence et distance requises par la technique considérée... Cela permet notamment d'éviter les phénomènes de sur ou sous-exposition rendant les radiogrammes non recevables.

Sur ce point, quelques entreprises de contrôles non destructifs (CICO Centre, Fig. 11 ; GIE Horus, Fig. 12, Institut de soudure...) ont développé des outils faciles à mettre en œuvre sur certaines configurations (soudures bout à bout, joints emmanchés soudés, techniques plan/plan en général...) (Fig. 11). Ils sont généralement constitués de rallonges métalliques réglables, de bagues de divers diamètres, d'un dispositif de visée laser ainsi que dans certains cas, d'un système d'atténuation des rayonnements...

\section{Axe d'amélioration}

Il est encouragé l'emploi, autant que faire se peut, de systèmes de positionnement source d'irradiation-objet contrôlé-film. En effet, tout en garantissant des bonnes conditions de mise en œuvre (respect des paramètres de la technique considérée), ces systèmes permettent aussi indirectement la réduction de l'exposition des opérateurs et un gain de temps pouvant être non négligeable (limitation des radiogrammes à refaire).

L'appareillage utilisé doit toutefois rester simple à mettre en œuvre.

\subsubsection{Radionucléides utilisés en sources scellées}

L'iridium-192 et le cobalt-60 monopolisent actuellement le marché français des contrôles non destructifs par gammagraphie. Mais depuis peu (janvier 2006), le sélénium-75 est aussi autorisé à être chargé dans les GAM80/120 fournis par Cegelec, à hauteur de 2,96 TBq. 


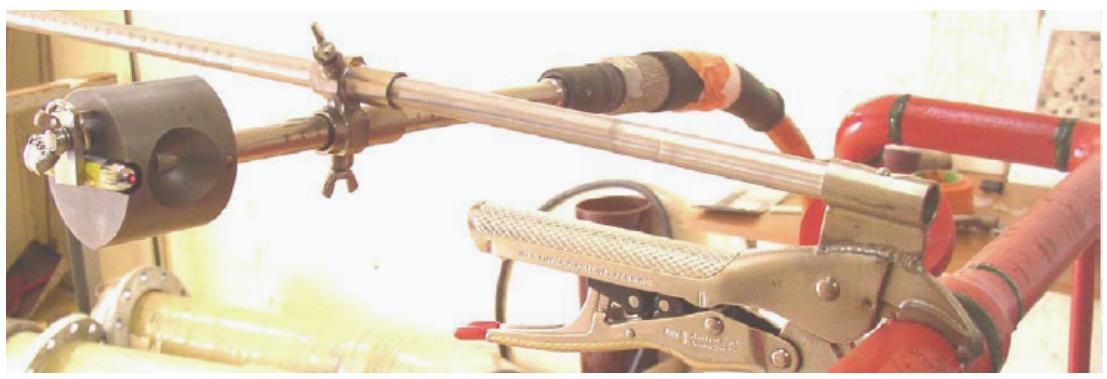

Figure 11 - Système CICO Centre ${ }^{\circledR}$. CICO ${ }^{\circledR}$ System of positioning.

D'un point de vue radioprotection, pour une même activité mise en œuvre, grâce aux rayonnements gamma du sélénium-75 en moyenne moins énergétiques que ceux du cobalt-60 et de l'iridium-192, la zone d'opération à définir lors des chantiers sera réduite (distances de balisage raccourcies d'environ $30 \%$ par rapport à l'iridium-192). Elle sera ainsi plus simple à délimiter et à surveiller, induisant notamment une amélioration de la maîtrise de la co-activité à proximité des chantiers de tirs gammagraphiques.

Par ailleurs, l'utilisation des collimateurs prévus pour l'iridium-192 atténuera plus fortement les rayonnements gamma du selenium-75.

L'emploi de ce radionucléide assure également une meilleure qualité d'image, se rapprochant de celle obtenue avec un appareil électrique générateur de rayons X.

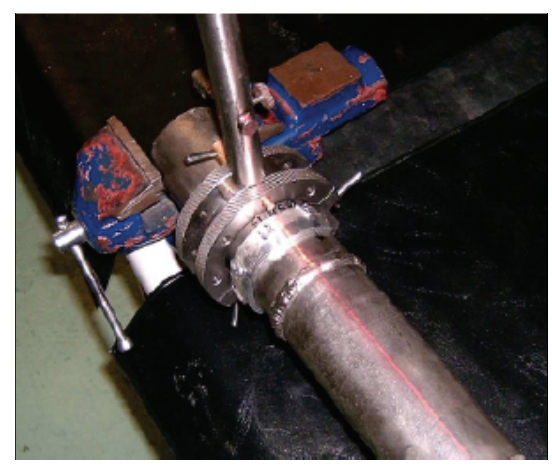

Figure 12 - Système GIE Horus ${ }^{\circledR}$.

GIE Horus ${ }^{\circledR}$ System of positioning. 
En contrepartie, pour une même épaisseur de matériau à contrôler, la durée d'exposition optimale des films sera plus longue qu'avec une source d'iridium-192.

On peut néanmoins penser que l'utilisation en France du sélénium-75, dont l'emploi semble assez répandu en Europe, pourrait prendre place entre les appareils générateurs électriques de rayons $\mathrm{X}$, souvent peu pratiques à mettre en œuvre sur chantiers, et l'iridium-192, mieux adapté au contrôle de pièces métalliques de plus « grandes » épaisseurs.

Pour rappel, le tableau II présente les gammes d'épaisseurs traversées (acier et alliages de cuivre et de nickel) pour chacun des radionucléides autorisés en France ${ }^{(1)}$.

\section{Propositions}

Pour les avantages qu'il procure en terme de réduction de la zone d'opération (facilité de surveillance, maîtrise accrue de la co-activité...), l'emploi du sélénium-75 en remplacement de l'iridium-192 est recommandée lorsque l'épaisseur de matériau à traverser et le type de défaut recherché le permettent.

Par ailleurs, afin d'en favoriser une utilisation plus large, les membres de l'atelier jugent nécessaire la mise à jour des codes, normes, procédures et modes opératoires de contrôle non destructifs ne prenant pas encore en compte le sélénium-75, notamment dans le domaine des équipements sous pression.

Au préalable de ces mises à jour, si besoin est, des études complémentaires d'optimisation des paramètres de mise en œuvre du sélénium-75 devraient être menées.

\subsection{Contrôles radiographiques à poste fixe (local dédié) ou dans un lieu régulier}

Pour s'affranchir des problématiques inhérentes aux contrôles radiographiques sur chantiers, tels la maîtrise de la co-activité, l'adaptation forcée à l'environnement

TABLEAU II

Les épaisseurs traversées.

Crossed thicknesses.

\begin{tabular}{lcccc}
\hline $\begin{array}{c}\text { Radionucléide } \\
\text { (par ordre décroissant d'énergie gamma moyenne) }\end{array}$ & cobalt-60 & iridium-192 & sélénium-75 \\
\hline épaisseur traversée pour un essai de classe A, en cm & de 4 à 20 & de 2 à 10 & de 1 à 4 (2) \\
épaisseur traversée pour un essai de classe B, en cm & de 6 à 15 & de 2 à 9 & de 1,4 à 4 \\
\hline
\end{tabular}

(1) Valeurs issues de la norme NF EN 1435 d'octobre 1997 (contrôle non destructif des assemblages soudés - contrôle par radiographie des assemblages soudés / Additifs A1 de juin 2003 et A2 de juillet 2004). Pour mémoire, au sens de la norme, la classe $\mathrm{A}$ correspond à des techniques radiographiques de base, la classe $\mathrm{B}$ a des techniques améliorées.

${ }^{(2)}$ Pour l'aluminium et le titane, l'épaisseur traversée est de 3,5 à $12 \mathrm{~cm}$ pour la classe A. 
rencontré ou la limitation de l'exposition des opérateurs, des locaux réservés exclusivement à ces contrôles peuvent être définis. L'appareil de radiographie sera alors considéré à poste fixe.

Il existe des normes d'installations relatives à l'aménagement de tels locaux, aussi bien pour la gammagraphie (norme NF M 62-102 de septembre 1992) que pour la radiographie X (norme NF C 15-160 de novembre 1975 + Additif A1 de septembre 1984 + NF C 15-164 de novembre 1976). Elles recommandent ou imposent (cas de la radiographie $\mathrm{X}$ ) certaines dispositions de construction et de sécurité :

- Pour la radiographie X: présence d'une double signalisation lumineuse spécifique aux accès du local, contacteurs de sécurité de porte asservis à l'émission des rayons $\mathrm{X}$, coup de poing d'arrêt d'urgence à l'intérieur du bunker, dispositifs spécifiques de protection du circuit d'alimentation du générateur haute tension...

- Pour la gammagraphie : présence d'une balise de mesure des rayonnements, coffret spécifique renfermant la télécommande mécanique, circuit spécifique de sécurité en cas de télécommande électrique, système de verrouillage des accès, signalisation sonore et lumineuse interne et externe de mise en service...

Par ailleurs, certains appareils de radiographie sont régulièrement utilisés dans les locaux d'ateliers de fabrication (tuyauterie, chaudronnerie...) avec une mise en œuvre de type « chantier ». Ces conditions peuvent parfois conduire à une mauvaise définition, et donc délimitation, de la zone d'opération (cf. paragraphe 2.3.1).

\section{Propositions}

Pour l'utilisation de gammagraphes à poste fixe dans un local dédié, il est proposé d'appliquer strictement la norme NF M 62-102 lors de la conception de nouvelles enceintes. Pour les installations existantes ne répondant pas aux attendus de la norme, il est recommandé de s'en rapprocher le plus possible.

En règle générale, de par leur niveau optimal de sécurité, les contrôles radiographiques en locaux dédiés sont à privilégier aux interventions de type « chantiers » lorsque les pièces à contrôler sont facilement déplaçables.

Dans le cas particulier de la mise en œuvre régulière d'opérations de radiographie industrielle dans une société « utilisatrice » (tuyauteurs, ateliers de chaudronnerie...), il est recommandé de favoriser l'aménagement d'une enceinte dédiée ou, a minima, d'adopter des dispositions matérielles pérennes (arrangements de murs en béton, zones semi-casematées...) permettant de limiter l'ambiance radiologique dans et hors des locaux concernés. 


\subsection{Matériels associés aux contrôles radiographiques}

\subsubsection{Délimitation et signalisation de la zone d'opération}

L'arrêté « zonage » du 15 mai 2006 introduit la notion de zone d'opération pour les appareils mobiles ou portables utilisés sur chantiers : cette zone doit notamment être délimitée de manière continue et signalée par des panneaux installés de manière visible. Cette signalisation doit mentionner la nature du risque et l'interdiction d'accès à toute personne non autorisée.

L'arrêté précise aussi qu'un dispositif lumineux doit être activé durant la période d'émission des rayonnements ionisants; il est complété en tant que de besoin par un dispositif sonore.

La réglementation actuelle stipule par ailleurs que l'accès au chantier doit être matériellement interdit pendant la durée de l'exposition par la mise en place de dispositifs ne pouvant être franchis par inadvertance.

Bien qu'une multitude de matériels de délimitation et de signalisation soit disponible sur le marché, les membres de l'atelier ont souhaité présenter les dispositifs ci-dessous du fait de leur concept novateur ou de leur aspect pratique.

Cette balise (Fig. 13), destinée à être placée à proximité de la source de rayonnements ionisants, est asservie au débit d'équivalent de dose ambiant. Elle comporte un seuil d'alarme automatique calculé en fonction du bruit de fond (déclenchement pour toute variation de $200 \mu \mathrm{Sv}^{-\mathrm{h}^{-1}}$ en moins d'une seconde) ainsi qu'un seuil fixe à $2 \mathrm{mSv} \cdot \mathrm{h}^{-1}$. L'alarme donnée est sous forme lumineuse et sonore.

L'autonomie garantie par le constructeur est de 26 heures en clignotement permanent.

Pour la gammagraphie, l'avantage de ce dispositif est qu'il donne de précieuses informations sur la présence réelle de la source radioactive hors du projecteur.

Néanmoins, afin de garantir une efficacité optimale de cette balise et outre le fait de s'assurer avant chaque utilisation de son niveau de charge (présence d'un témoin «batterie faible »), il est souhaitable de faire vérifier périodiquement le bon fonctionnement du système intégré de mesure des rayonnements ionisants.

Ces panneaux (Fig. 14) sont destinés à renforcer le signalement des limites de la zone d'opération. Des diodes clignotantes sont placées au cœur du trèfle et à l'extrémité de chacune de ses « feuilles », attirant ainsi l'attention.

Un capteur (faces avant et arrière, rayon de détection : 5 mètres) est proposé en option : en face avant, les diodes lumineuses se déclenchent en cas de détection 


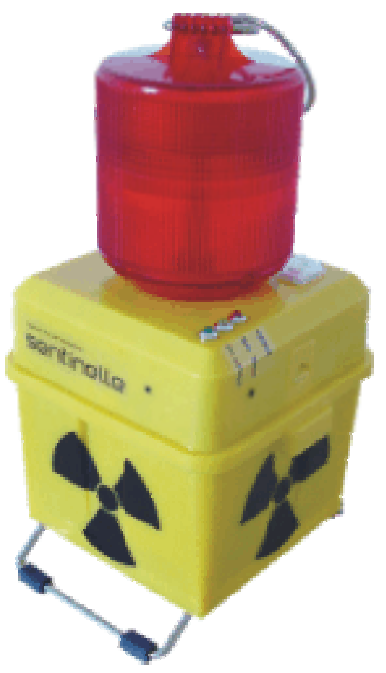

Figure 13 - Balise "Sentinelle " ${ }^{\circledR}$ (Sté CARMELEC). Mark Out "Sentinelle" ${ }^{\circledR}$ (CARMELEC Cie).

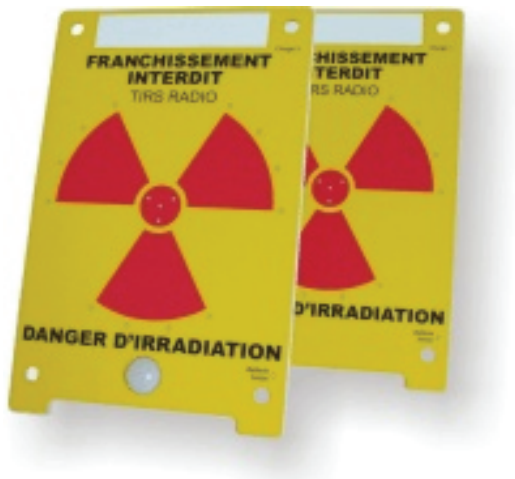

Figure 14 - Panneaux lumineux d'avertissement (Sté CARMELEC). Luminous panels of warning (CARMELEC Cie). 
de présence, et en face arrière, le signal lumineux est complété par une alarme sonore.

L'autonomie constatée est de deux à trois jours.

\section{Bandes de délimitation / fixation associée}

La norme NF M 60-103 de juillet 1976, concernant les bandes de balisage pour la délimitation de zones particulières pouvant présenter des risques radiologiques, prescrit l'utilisation de bandes normalisées de $10 \mathrm{~cm}$ de large, la distance séparant deux motifs (« trèfle radioactif ») étant de $60 \mathrm{~cm}$ (Fig. 15).

Les couleurs retenues pour signaler le franchissement interdit à toute personne non autorisée sont les suivantes : trèfles blancs, fond rouge, lettres noires (en majuscules de $2 \mathrm{~cm}$ de hauteur).

Plusieurs sociétés commercialisent ce type de bandes en rouleau de diverses longueurs ou même en longueur réduite avec œillets pour en faciliter la fixation.

Les opérateurs sont d'ailleurs souvent confrontés à ce type de problèmes : adhésifs qui se décollent, piquets de fixation trop légers...

Sur ce point, quelques sociétés proposent la personnalisation de poteaux à sangle de type Beltrac (12 kg par poteau, sangle de 2 à 4,5 m) (Fig. 16). Cela peut constituer une solution pour le balisage de petites surfaces ou d'entrée de locaux. Une version murale fixe existe également : elle serait intéressante pour des contrôles réguliers effectués dans un même lieu dans le cas où l'aménagement d'une enceinte dédiée ne serait pas possible (cf. paragraphe 2.2).

\section{Dispositif de surveillance globale de la zone d'opération}

Un dispositif prototype, conçu par MGP Instruments, est actuellement en phase de test chez EDF. Ce système, baptisé PRI (Protection des Radiographies Industrielles), est notamment constitué d'une balise de surveillance spécifique (alarmes lumineuses et sonores, mesure du débit d'équivalent de dose ambiant, capteurs de présence, transmission radio) placée à proximité de la source de rayonnements ionisants et de modules de réception à la disposition des opérateurs.

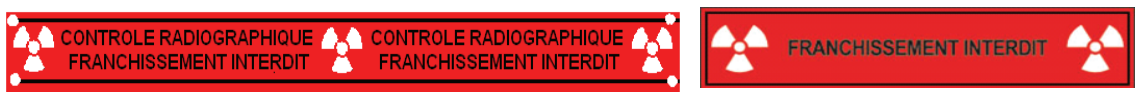

Figure 15 - Bandes normalisées.

Standardised bands. 


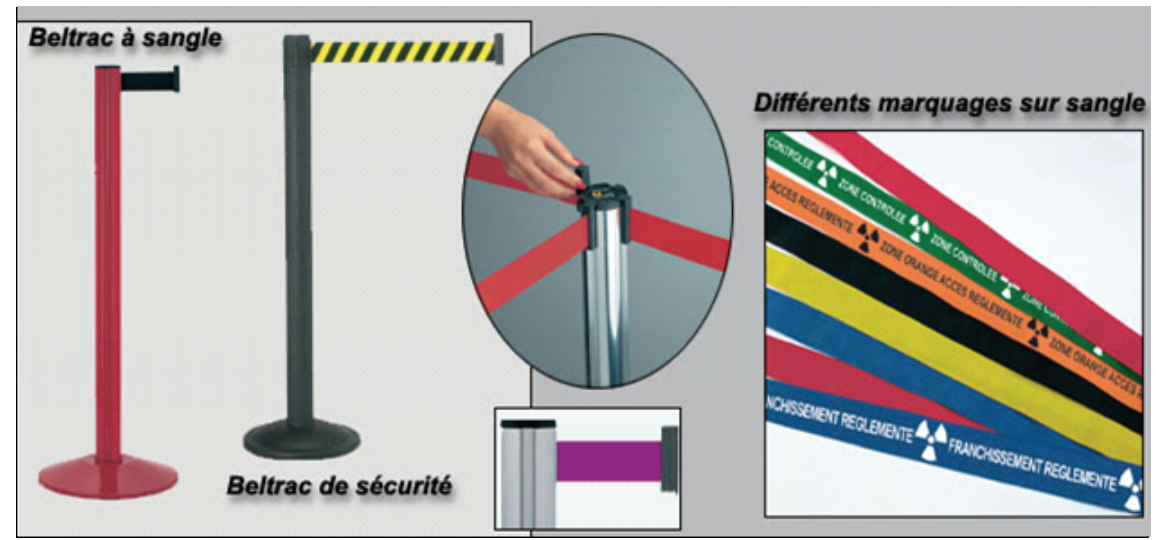

Figure 16 - Sangles BELTRAC.

BELTRAC straps.

Ce système est conçu pour que toute intrusion dans la zone dite « de défense » (approximativement une sphère de $15 \mathrm{~m}$ de rayon autour de la balise) soit détectée et portée à la connaissance de l'opérateur via une alarme sur le module de réception.

Ce dispositif, bien qu'intéressant dans l'absolu, doit cependant être validé en situation réelle de fonctionnement. Son coût sera également à prendre en compte.

\subsubsection{Protections collectives}

Dans le cas où l'emploi d'un collimateur s'avèrerait impossible lors d'un contrôle gammagraphique (cf. paragraphe 2.1.3), des moyens simples (Fig. 17)

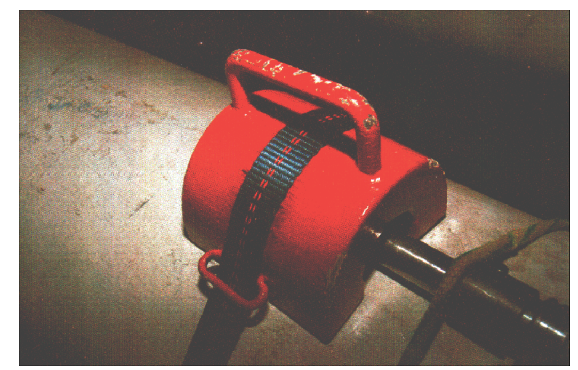

Figure 17 - Cloche métallique adaptée à certaines tuyauteries.

Metal bell adapted to certain pipings. 


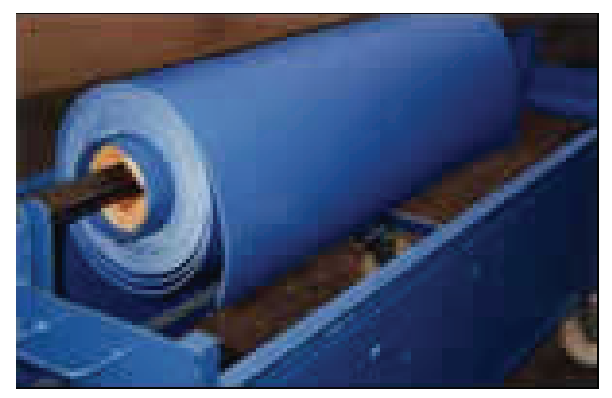

Figure 18 - GammaStop ${ }^{\circledR}$ (Sté IDEALEX). Gammastop $^{\circledR}$ (IDEALEX Cie).

de protection collective (matelas de plomb...) peuvent être utilisés pour réduire significativement l'ambiance radiologique et par conséquent, limiter la zone d'opération. Néanmoins, des protections adaptées, en acier ou en plomb, peuvent également être manufacturées pour une géométrie donnée.

Les membres de l'atelier ont par ailleurs choisi de présenter les dispositifs ci-dessous du fait de leur ingéniosité ou de leur facilité de mise en œuvre sur chantiers.

\section{GAMMASTOP (sté IDEALEX)}

C'est une protection radiologique (Figs. 18 et 19) souple de $5 \mathrm{~mm}$ d'épaisseur que l'on peut acquérir sous forme de rouleau, de matelas simple ou double épaisseur (avec aillets de suspension), de pièces moulées ou prédécoupées...

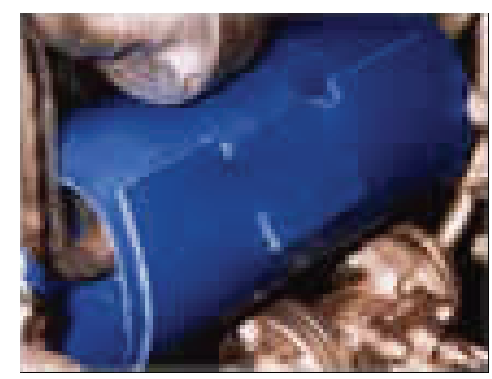

Figure 19 - GAMMASTOP ${ }^{\circledR}$ appliqué sur un tuyau. GAMMASTOP $^{\circledR}$ applied to a pipe. 
Le matériau utilisé est un élastomère chargé au plomb; sa masse surfacique est de $15 \mathrm{~kg} . \mathrm{m}^{-2}$, sa densité de 3 . Il se découpe au cutter et peut donc s'adapter facilement aux diverses configurations rencontrées dans les chantiers.

Les épaisseurs moitié données par le fabricant sont les suivantes : $45 \mathrm{~mm}$ pour le cobalt-60 et $15 \mathrm{~mm}$ pour l'iridium-192.

\section{GammaBlok (Sté ZaDA Technologies, Sté SAFERAD)}

Le GAMMABLoK (Fig. 20) est également une protection radiologique souple que l'on peut acheter en différentes dimensions (épaisseurs standards : 8 et $15 \mathrm{~mm}$ ). Elle est facilement découpable au cutter et peut donc ainsi s'adapter à la configuration recherchée.

Son matériau constitutif ne contient ni plomb ni métaux toxiques. Sa densité annoncée est de 4,97.

Les épaisseurs moitié annoncées par le fabricant sont les suivantes : $6 \mathrm{~mm}$ pour le sélénium-75, $11 \mathrm{~mm}$ pour l'iridium-192 et $28 \mathrm{~mm}$ pour le cobalt-60.

\subsubsection{Matériels de détection / Suivi dosimétrique opérationnel}

Pour rappel, lors de l'utilisation d'un gammagraphe, la position de la source radioactive au moment de l'armement et le retour de celle-ci dans le projecteur en position de sécurité doivent être vérifiés systématiquement lors de chaque opération au moyen d'un appareil de mesure des rayonnements ionisants, de type radiamètre, adapté à la plage d'énergie des rayonnements considérés.

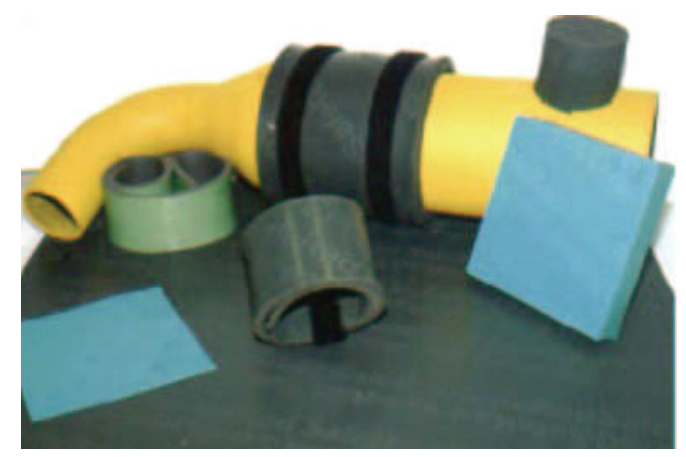

Figure 20 - GAMMABLOK ${ }^{\circledR}$ (Sté ZADA Technologies, Sté SAFERAD).

GammaBlok $^{\circledR}$ (ZADA Technologies Cie, SAFERAD Cie). 
Par ailleurs, au regard de l'arrêté du 30 décembre 2004 relatif aux informations individuelles de dosimétrie des travailleurs exposés, les dosimètres opérationnels portés par les travailleurs susceptibles d'intervenir dans la zone d'opération doivent être munis de dispositifs d'alarme, visuels et/ou sonores, permettant ainsi d'informer leur porteur en cas de dépassement du seuil de débit de dose ou de dose cumulée, réglé au préalable de l'opération.

\section{Axes d'amélioration}

Il est préconisé le port de dosimètres opérationnels à alarmes visuelles et sonores lors de tout contrôle de radiographie industrielle.

De plus, à l'instar des technologies utilisées pour la téléphonie portable, la présence d'une alarme de type vibratoire sur ce type de matériel peut s'avérer intéressante. En effet, suivant les conditions d'intervention (ambiance sonore élevée, port de tenues masquant ou atténuant le signal lumineux) et la fatigue éventuelle de l'opérateur, l'alarme vibratoire pourrait représenter une barrière de sécurité supplémentaire de choix.

\section{Contrôle, vérification et suivi des appareils et de leurs accessoires}

\subsection{Contrôles techniques réglementaires}

Au regard de l'arrêté du 26 octobre 2005 définissant les modalités de contrôle de radioprotection, les contrôles techniques de radioprotection à effectuer sur les appareils de radiographie industrielle sont les suivants :

- contrôle externe annuel par un organisme agréé ou l'IRSN,

- contrôle interne semestriel pour les appareils électriques générateurs de rayons X utilisés sur chantiers,

- contrôle interne trimestriel pour les gammagraphes (car contenant une source radioactive de haute activité, au sens de l'annexe 13-7 de la première partie du Code de la santé publique : activité initiale supérieure à $10 \mathrm{GBq}$ pour l'iridium-192, 4 GBq pour le cobalt-60 et 30 GBq pour le sélénium-75).

\subsection{Maintenance et révisions périodiques}

Le décret $n^{\circ} 85-986$ impose une révision a minima annuelle (sauf prescription plus contraignante sur la notice d'instruction « fournisseur ») des gammagraphes et de leurs accessoires pour ceux où le porte-source et le dispositif d'éjection sont liés 
mécaniquement. Ces révisions doivent être exécutées par des techniciens dûment qualifiés sous la responsabilité du constructeur ou de son représentant français.

\section{Proposition}

La date de révision annuelle de l'appareil est souvent très proche de sa date de rechargement, dans le cas de sources d'iridium-192. Ainsi, afin de limiter les transports (un pour le rechargement et un pour la révision) et laisser plus de souplesse aux entreprises quant à leur planning de contrôles, il est proposé d'introduire une tolérance de 6 semaines sur la date « anniversaire » de révision d'un appareil pour permettre, le cas échéant, d'effectuer simultanément son rechargement. Néanmoins, la prochaine révision de l'appareil restera toujours prévue à la date « anniversaire » (avec, donc, une tolérance de 6 semaines sur la réalisation effective de la révision).

\subsection{Documents de suivi}

Un carnet ou registre de suivi doit accompagner chaque gammagraphe. Son contenu et ses règles d'utilisation sont fixés dans l'arrêté du 11 octobre 1985 : identification du gammagraphe et de son détenteur, enregistrement des chargements successifs, des paramètres d'exploitation, des contrôles réglementaires, des opérations de maintenance...

Sur le même principe, une fiche de suivi doit accompagner chaque accessoire (télécommande, gaine d'éjection, embout d'irradiation et collimateur).

\section{Proposition}

Pour chaque chantier, les paramètres d'exploitation à noter sur le carnet de suivi du gammagraphe sont les suivants : lieu et nombre d'éjection de la source, nom de l'opérateur, date d'obtention de son CAMARI, identification des accessoires utilisés, anomalies de fonctionnement constatées et décisions consécutives.

Du fait de la lourdeur que représente lors de chaque chantier la consignation de ces paramètres par l'opérateur, et eu égard au fait qu'ils ne sont vraisemblablement exploités par personne (autorités, constructeur, utilisateurs), il est proposé de supprimer l'enregistrement systématique des paramètres d'exploitation.

\section{Synthèse et conclusion de l'atelier « Matériel et exigences associées »}

Cette section récapitule les divers axes d'améliorations et propositions retenus par les membres de l'atelier. 


\subsection{Axes d'amélioration}

- Pour les projecteurs gammagraphiques de type GAM80/120 :

- amélioration de l'ergonomie de la poignée,

- ajout de points d'élingue amovibles,

- renforcement de la visibilité de la signalisation colorée.

- Ajout d'une barrière physique supplémentaire (clapet, membrane) sur les embouts des différentes gaines (éjection et télécommande).

- Substitution de l'uranium appauvri des collimateurs par des alliages à base de tungstène.

- Mise en ouvre de systèmes de positionnement source d'irradiation-objet contrôlé-film.

- Port par les opérateurs de dosimètres opérationnels à alarmes visuelles et sonores lors de tout contrôle de radiographie industrielle.

La présence d'une alarme vibratoire sur ce type de matériel serait à envisager.

\subsection{Propositions}

- Emploi systématique lors de chantiers gammagraphiques d'un dispositif de collimation ou d'atténuation du rayonnement, sauf impossibilités techniques avérées (où la mise en œuvre de dispositifs adaptés de protection collective doit alors être recherchée).

Révision des codes, procédures, normes ou modes opératoires relatifs aux contrôles non destructifs pour tenir compte de cette systématisation.

- Remplacement de l'iridium-192 par le sélénium-75 lorsque l'épaisseur de matériau à traverser et le type de défaut recherché le permettent.

Modification des codes, procédures, normes ou modes opératoires pour y intégrer l'emploi du sélénium-75.

- En cas d'utilisation de gammagraphes à poste fixe dans un local dédié, application stricte de la norme NF M 62-102 lors de la conception de nouvelles enceintes. Pour les installations existantes, l'exploitant devra se rapprocher le plus possible des attendus de la norme.

Les contrôles radiographiques en locaux dédiés doivent être privilégiés aux interventions de type « chantiers » lorsque les pièces à contrôler sont facilement transportables.

Lorsque des contrôles radiographiques sont régulièrement mis en œuvre dans une société " utilisatrice » (de type ateliers de chaudronnerie, de tuyauterie...), 
l'aménagement d'une enceinte dédiée ou, a minima, l'adoption de dispositions matérielles pérennes visant à réduire l'ambiance radiologique est préconisée.

- Introduction d'une tolérance de six semaines sur la date de révision annuelle des appareils de gammagraphie et de leurs accessoires (la date de révision restant par ailleurs calée sur une périodicité de douze mois, sans tenir compte de la tolérance).

- Suppression de l'enregistrement systématique des paramètres d'exploitation (lieu et nombre d'éjections de la source, nom de l'opérateur...) sur le carnet de suivi du gammagraphe concerné.

\subsection{Conclusion}

Les membres du présent atelier se sont efforcés, d'une part, de dégager plusieurs axes d'amélioration quant au matériel, au sens large, utilisé lors des contrôles radiographiques et, d'autre part, de proposer des dispositions d'ordre organisationnelles ou techniques permettant de faire évoluer les pratiques actuelles vers une meilleure radioprotection ou sécurité des opérateurs et de leur environnement. Il a été par ailleurs choisi de focaliser les réflexions sur le matériel utilisé en gammagraphie ; les problématiques liées à la mise en œuvre d'appareils électriques générateurs de rayons $\mathrm{X}$ n'ont cependant pas été exclues.

En premier lieu, la réalisation de contrôles radiographiques dans des locaux réservés exclusivement à cet effet doit être privilégiée. Ces enceintes présentent théoriquement des garanties de sécurité et de radioprotection plus élevées qu'une mise en œuvre de type «chantier », pour peu qu'elles soient réalisées dans les règles de l'art. En particulier, toute nouvelle enceinte de gammagraphie devrait être conçue suivant la norme NF M 62-102 (ce n'est pas aujourd'hui une obligation réglementaire). L'atelier propose également que les installations existantes soient modifiées pour tendre autant que raisonnablement possible vers les attendus de la norme précitée.

Le cas échéant, pour des contrôles radiographiques réguliers dans un même lieu (ateliers de fabrication, par exemple), les membres de l'atelier insistent sur la nécessité de prendre des dispositions matérielles pérennes afin de limiter l'ambiance radiologique dans et hors des locaux pendant ces contrôles.

Bien que certaines choses soient plus ou moins figées (réglementairement ou commercialement), une évolution du matériel utilisé sur chantiers est potentiellement envisageable à long terme : projecteurs gammagraphiques, gaines, collimateurs, dosimètres opérationnels... Les pistes d'amélioration identifiées devraient permettre entre autres d'améliorer les conditions d'intervention des opérateurs. 
De plus, pour limiter l'exposition de ces derniers lors des contrôles, les membres de l'atelier proposent l'utilisation systématique de dispositifs de collimation ou d'atténuation du rayonnement (ou, si impossibilités, d'avoir recours à des moyens appropriés de protection collective), ainsi que la généralisation de l'emploi de gammagraphes chargés au sélénium-75 (au lieu d'iridium-192) lorsque les conditions le permettent.

Concernant la délimitation/signalisation des zones d'opération et les protections collectives, plusieurs matériels innovants sont déjà disponibles sur le marché. Les membres de l'atelier ont voulu mettre en valeur certains d'entre eux, du fait de leur aspect pratique ou du gain apporté en matière de radioprotection/ sécurité, afin de les porter à connaissance du plus grand nombre.

Par ailleurs, diverses initiatives actuelles d'entreprises œuvrant dans la radiologie industrielle méritent d'être soulignées et encouragées : conception d'atténuateurs en alliage de tungstène, mise au point de divers systèmes de positionnement source-objet-film...

En conclusion, au vu des échanges au sein du présent atelier et du retour d'expérience issu du « terrain », l'évolution ou l'optimisation du matériel aujourd'hui utilisé lors des contrôles radiographiques participera grandement à l'amélioration de la sécurité et de la radioprotection des opérateurs, sans alourdir leurs conditions d'intervention (bien au contraire).

Il faut cependant veiller à ce que ces progrès, actuellement en cours, ne s'essoufflent pas. Pour cela, ils doivent être nécessairement partagés et soutenus par l'ensemble des acteurs concernés : radiologues, entreprises de contrôles non destructifs, « donneurs d'ordre », constructeurs/fournisseurs d'appareil et autorités administratives. 